\title{
Uptake of three doses of HPV vaccine by primary school girls in Eldoret, Kenya; a prospective cohort study in a malaria endemic setting
}

Hillary Mabeya ${ }^{1,2^{*}}$, Sonia Menon ${ }^{2,3}$, Steven Weyers ${ }^{4}$, Violet Naanyu' ${ }^{1}$, Emily Mwaliko ${ }^{1,2}$, Elijah Kirop ${ }^{1}$, Omenge Orango ${ }^{1}$, Heleen Vermandere ${ }^{2}$ and Davy Vanden Broeck ${ }^{2}$

\begin{abstract}
Background: All women are potentially at risk of developing cervical cancer at some point in their life, yet it is avoidable cause of death among women in Sub- Saharan Africa with a world incidence of 530,000 every year. It is the 4th commonest cancer affecting women worldwide with over 260,000 deaths reported in 2012. Low resource settings account for over $75 \%$ of the global cervical cancer burden. Uptake of HPV vaccination is limited in the developing world. WHO recommended that 2 doses of HPV vaccine could be given to young girls, based on studies in developed countries. However in Africa high rates of infections like malaria and worms can affect immune responses to vaccines, therefore three doses may still be necessary. The aim of this study was to identify barriers and facilitators associated with uptake of HPV vaccine.
\end{abstract}

Methods: A cross-sectional survey was conducted at Eldoret, Kenya involving 3000 girls aged 9 to 14 years from 40 schools. Parents/guardians gave consent through a questionnaire.

Results: Of all 3083 the school girls 93.8\% had received childhood vaccines and 63.8\% had a second HPV dose, and 39. $1 \%$ had a third dose. Administration of second dose and HPV knowledge were both strong predictors of completion of the third dose. Distance to the hospital was a statistically significant risk factor for non-completion (P: 0.01).

Conclusions: Distance to vaccination centers requires a more innovative vaccine-delivery strategy and education of parents/guardians on cervical screening to increase attainment of the HPV vaccination.

Keywords: Kenya, Gardasil, HPV vaccination, Determinants, Parents/guardians, Malaria

\section{Background}

All women are potentially at risk of developing cervical cancer at some point in their life, yet it is avoidable cause of death among women in Sub- Saharan Africa with a world incidence of 530,000 every year. It is the 4th commonest cancer affecting women worldwide with over 260,000 deaths reported in 2012. Low resource settings account for over $75 \%$ of the global cervical cancer burden [1].

\footnotetext{
*Correspondence: mabeya4@gmail.com; icrh@ugent.be

${ }^{1}$ Moi University, Eldoret, Kenya

${ }^{2}$ International Center of Reproductive Health, Ghent University, De Pintelaan 185 P3, 9000 Ghent, Belgium

Full list of author information is available at the end of the article
}

Cervical cancer remains the most common cancer in Eastern and Central Africa with an estimated with estimated age standardized rates (ASRs) of 42.7 and 30.6 respectively compared with 2-5 in Western Europe and Australia. Mortality is highest in Eastern Africa at 27 per 10,000 compared to less than 2 per 100,000 in Western Asia/Europe, Australia and New Zealand [1].

Among the Eastern African countries, Kenya has a high cervical cancer burden which is the leading cause of cancer deaths in women of reproductive age and the 2nd most frequent cancer among women in Kenya. The incidence of cervical cancer cases in Kenya is estimated at close to 2500 with annual deaths close to just over 1500 with an average life lost estimated at 25 years. The

(c) The Author(s). 2018 Open Access This article is distributed under the terms of the Creative Commons Attribution 4.0 International License (http://creativecommons.org/licenses/by/4.0/), which permits unrestricted use, distribution, and reproduction in any medium, provided you give appropriate credit to the original author(s) and the source, provide a link to the Creative Commons license, and indicate if changes were made. The Creative Commons Public Domain Dedication waiver (http://creativecommons.org/publicdomain/zero/1.0/) applies to the data made available in this article, unless otherwise stated. 
incidence is projected to double by the year 2025 if no efforts are put to mitigate the increment. Secondary prevention for women in reproductive age is only $3 \%$ compared with $40-70 \%$ in high resource countries [2-6].

HPV vaccination as a primary prevention method is supplemented by screening later in life. This is because vaccines do not protect against all high-risk and potential high-risk HPV types. Vaccines to prevent cervical cancers are now available. The quadrivalent (Gardasil 4) vaccine protecting against human papilloma virus (HPV) types $16 / 18 / 6 / 11$ and the bivalent (Cervirax) vaccine protecting against infection with HPV-16/18 were both originally used as a three dose schedule. A third vaccine, the nonavalent HPV (Gardasil 9) vaccine protecting against HPV types 16/18/6/11/31/33/45/52/58 has been approved by Food and Drug Administration (FDA) in 2014 in the USA [7]. The cost and logistical difficulties and cost in resource-constraint settings has delayed or slowed implementation of the original three-dose vaccine regimen. GAVI Alliance supported HPV vaccination in 2011 and if less than three dose schedule is adopted in these countries, there will be an increase the implementation of HPV vaccination programs in resourceconstraint countries $[4,7]$. Three vaccines are now being marketed in many countries as primary prevention intervention tool. HPV 16 and 18 are responsible for approximately $70 \%$ of cervical cancer cases globally. Gardasil and Nonavalent also prevent anogenital warts that are caused by HPV 6 and 11 [8].

World Health Organisation has recommended offering HPV vaccination to girls at ages 9-13 and Kenya has developed a comprehensive strategy to address cervical cancer, which includes rollout of HPV vaccination among girls, with the purpose of vaccinating girls naïve to the targeted types. This roll out of HPV vaccine among girls naive to the targeted types, is akin to the one which took place in Rwanda, Uganda, Cameroon, Tanzania, Lesotho and South Africa, which have introduced HPV vaccine to prevent cervical cancer through school-based programme [5-7, 9, 10, 11].

WHO has recommended bivalent or quadrivalent vaccine in the 2-dose schedule with a 6-month interval between doses for females less than 15 years old, including females 15 years or older at the time of the second dose based on available studies, before the females become sexually active. A third dose is recommended at least 6 months after the first dose, if the interval between the two doses is shorter than 5 months and for 15 years old or more who are known to be immunocompromised and/or HIV-infected or are already sexually active. This 2 dose recommendation will lead to cost saving and other logistic problems of delivering the vaccine and compliance $[2,7]$.

Education and knowledge by health personnel and parents/guardians about cervical cancer, HPV and HPV vaccines is necessary for vaccine uptake and hence enhanced primary prevention of cervical cancer [12-15]. An HPV immunization program must also consider the unique challenges that HPV vaccination implementation faces, including a recommended schedule consisting of 2 doses for 9 to 14 year old girls and 3 doses for those above 15 years and if they are immunocompromised. So far, there is no published research from Kenya on risk factors for partial HPV regimen administration.

This study sought to explore barriers and facilitators that undermine or promote adherence to 3 doses HPV vaccine in a malaria and helminthic endemic western Kenya and explore policy implications for HPV vaccine introduction. Watson-Jones et al. in their attitudes and access to HPV vaccination among Maasai pastoralists and slum dwellers study found challenges to an HPV vaccination programme included early age of sex and marriage, lack of parental support, school absenteeism and drop-out, population mobility and distance from services. Despite little prior knowledge of cervical cancer and HPV, and school-based vaccination, communities were interested in receiving HPV vaccination. Adequate social mobilization supplemented by out-reach activities, were considered important facilitating factors to achieve high coverage [16].

HPV vaccines in populations whose immunological system may be challenged by multiple co-infections such as malaria and helminth infections are needed measure long term protection and advice on booster doses. Studies have shown that malaria and helminth infections have an effect on long term vaccine responses and hence more research is needed $[17,18]$.

In 2007 GAP reached more than 445,000 girls in 21 countries and several have implemented a national vaccination program. The burden of cervical cancer remains a big problem in developing world and the GAP initiative through the vaccine delivery will continue to play a valuable role as countries consider large-scale implementation. The demonstration programs and comprehensive analysis of the vaccine delivery has been published in BMC Public Health in May 2012 [19, 20].

In sub-Saharan Africa, many countries are considering the implementation of HPV vaccination. These countries have a high prevalence of endemic malaria and helminth infections that can act as immunological modulators and impact responses to standard immunizations. These infections can impact immune responses to vaccinations [21-27].

Sub-Saharan Africa except South Africa has high incidences of malaria. In Kenya, malaria remains a major cause of morbidity and mortality with more than $70 \%$ of the population at risk of the disease ( $\mathrm{MOH} 2014)$. More than four million cases of malaria are reported annually in Kenya. A 5.1\% mortality ratehas been reported among patients admitted with severe malaria. The malaria 
burden in Kenya is not homogeneous. Western Kenya, areas around Lake Victoria and on the cost present the highest risk [28-30].

A high HPV immunogenicity regardless of the presence of malaria and helminth infections among young girls and women in Tanzania study was found. There was some evidence of enhanced antibody titres to HPV vaccine genotypes in participants with malaria parasitaemia. Additional research on the impact of parasitic infection on the long-term duration of protection from $\mathrm{HPV}$ vaccines is warranted [31].

There is a paucity of information on the association between malaria and/or helminthic disease and cervical disease progression. The way malaria and helminths affect the immune response to HPV vaccine long term protection needs to be investigated. Studies should explore differences between levels of HPV 16/18 antibodies after administration of the vaccine among girls exposed to malaria/and or helminthic infections [32].

\section{Methods}

\section{Promotion}

Through the GARDASIL Access Program (GAP), the Moi Referral and Teaching Hospital (MTRH) was granted 9600 doses of the quadrivalent vaccine to administer to young girls in Eldoret, Kenya. MTRH is Kenya's second largest referral hospital with a bed capacity of 550 of which 100 are in reproductive health wards, with a service population of 13 million people. It is located in Eldoret town about $325 \mathrm{~km}$ from Nairobi in Western part of Kenya. It is a teaching hospital for both undergraduate and post graduate students from Moi University.

Eldoret is a principal city in western Kenya. It also serves as the capital of Uasin Gishu County. Lying south of the Cherangani Hills, the local elevation varies from about 2100-2700 (7000-9000 ft). The population was 289,380 in the 2009 census and it is currently the fastest growing town in Kenya.

Eldoret experiences highland malaria transmission if temperatures become warm and rainfall encourages mosquito breeding. Residents of Eldoret do not develop immunity and suffer much more serious illness than their lowland neighbours.

Promotion of the program was restricted to a number of randomly selected government primary schools, although other girls from the community were not refused if appeared at the vaccination site. A hospital-based vaccination was chosen to reduce the costs of the program.

The vaccines were offered to girls of age 9 to 14 years old, from ten primary schools in Eldoret Municipality. These schools were randomly selected until a total of about 4000 eligible girls was reached, expecting a coverage of around $75 \%$ (3000/4000). Permission to roll out the vaccination was given from the County Director of
Education who in turn instructed the head teachers of involved schools. A training of trainers (TOT) process was set in motion, with teachers first being trained by health staff and through the provision of leaflets the teachers were asked to instruct students and parents/ guardians on HPV vaccination and cervical cancer.

Vaccination took place from May 2012 to March 2013. After a signed consent was obtained from the parent/ guardian, nurses vaccinated the girls. A vaccination card with a next appointment was given upon the first and second dose. Additionally, caregivers who failed to show up on the schedule day were given a reminder by nurses about the second and third dose. Due to a low response during the first 3 months of the program, other schools in the County, government and private, were also invited to participate from August 2012 onwards through posters in the hospital and radio. In September 2012, the program stopped administering the first dose after reaching 3000 girls with a view to guaranteeing sufficient vaccines for the remaining doses.

\section{The survey}

This was done before administration of the vaccine. The questionnaire consisted of two parts. The first part collected information on sociodemographic characteristics, including religion and age of school girls. The second part consisted of questions regarding the guardian's knowledge of HPV, Pap smear knowledge and whether the female guardian/spouse had undergone a Pap smear test. The survey used in this study was designed by public health specialists and physicians. Data was collected from $22 / 04 / 2012$ to $23 / 04 / 2014$ by data collection team. The survey was pilot-tested by for comprehensibility before study commencement.

Interviewers administered questionnaires in English, and verbal interpretation in Swahili based on the participant's request.

\section{Data analysis}

Following data checking and cleaning, analysis was undertaken using STATA version 12. Corporation, College Station, TX, USA. In order to assess whether a median or mean should be given for continuous variables, a Shapiro-Wilk test at an alpha level of $5 \%$ was performed to assess whether the variable was parametric. We checked to see whether the missing data $(<5 \%)$ was missing completely at Random by performing the Little's MCAR test. Prob $>$ chi-square $=0.5472$ so indicating that the data missing is completely at random and is unlikely to have altered our results and multiple imputation is not required.

Dates for second dose, third dose, Pap smear undertaken, HPV knowledge was dichotomized into the two categories, yes and no. Google maps were used to 
calculate distance from School to vaccination center. Distance to health centre in kilometres and age were both used as a continuous variable and the nonparametric Mann Whitney rank sum test was performed as it could not be assumed that the continuous variables were normally distributed. Bivariate logistic regression was used to measure the strength of the association, with the dependent variable being the number of dose. Statistical analyses were performed using 'svy'command to account for clustering in making variance estimates, with school being the primary sampling unit.

A statistical test was considered significant if the $p$-value is 0.05 and borderline significant it was between 0.06 and 0.1 .

\section{Results}

\section{Population characteristics}

The median age range of schoolgirls whose caregivers were interviewed was 12 (IQR: 10-13), of which, 2853 (99.2\%) was Christian and $22(0.8 \%)$ Muslim. 843 (28. $1 \%)$ had a male parent, 2154 (71.8\%) had a female parent and $3(0.1 \%)$ had both. $786(27 \%)$ of parents/guardian had no knowledge of Pap tests. 307(14.4\%) of all female guardians underwent a Pap test; 824 (27.2\%) of parents/ guardians had knowledge of HPV. Of all the school girls eligible for this study 2808(93.8\%) had childhood vaccines and 1933(63.8\%) had second HPV dose, and 1182 (39.1\%) had a third dose. The mean time elapsed between the second HPV vaccine and the first vaccine was 32.5 days (SD 22.7), the third HPV vaccine and the second vaccine 148 days (SD: 17.5). The median time elapsed between the first HPV vaccine dose and the third HPV vaccine dose was 175 days (IQR: 168-182) (see Table 1).

\section{Barriers and facilitators of HPV vaccine uptake}

Administration of second dose of HPV and HPV knowledge were both strong predictors of taking the third dose of HPV vaccine (OR: $61.1 ; p<0.001 ; 95 \% \mathrm{CI}=40.9-91.0$ ) and (OR: $1.2 ; p=0.008 ; 95 \% \mathrm{CI} 1.1-1.5$ ) respectively. A Mann Whitney test revealed that distance to health facility was a statistically significant barrier (p.0.01).

A 14\% higher odds of administration of all three doses was found for girls who had a male guardian $(p=0.04$; CI: 1.0-1.3). A marginal association was observed between Pap test knowledge of the guardian and third HPV vaccine (OR: 1.2; p:0.08; CI:1.0-1.4).

No statistically significant risk factors were observed for childhood vaccine (OR: 1.2; p: 0.4; CI: 0.8-1.6), religion (OR:1.1; p: 0.9; CI: 0.5-2.5) and female guardian who had a Pap test OR 0.9; p:0.6; CI:0.7-1.2. A Mann Whitney rank sum test did not reveal a significant difference in age medians between those who had the third HPV vaccine and those who did not. (p: 0.1). (See Table 2).

\section{Discussion}

Three studies have been conducted in Kenya on actual HPV vaccination, our study and Ministry of Health GAVI HPV demonstration project in Kitui Kenya (2015).

Table 1 Population characteristics

\begin{tabular}{|c|c|c|}
\hline Population characteristics & Number & Percentage and Confidence Interval \\
\hline Age & 12 (IQR: 10-13) & \\
\hline \multicolumn{3}{|l|}{ Religion } \\
\hline Christian & $2853 / 2875$ & 99.20\% (95\%Cl: 99.0-99.5\%) \\
\hline Muslim & $22 / 2875$ & $0.80 \%(95 \% \mathrm{Cl} .0 .5-1.2)$ \\
\hline \multicolumn{3}{|l|}{ Caregiver } \\
\hline Female & $2154 / 3000$ & $71.8 \%$ (95\%Cl: 70.2-73.4) \\
\hline Male & $843 / 3000$ & $28.1 \%$ (95\% Cl: 26.5-29.7) \\
\hline \multicolumn{3}{|l|}{ Knowledge } \\
\hline Knowledge of Pap Smear tests & $786 / 2913$ & 27\% (95\%Cl: 25.4-28.6) \\
\hline HPV knowledge among caregivers & $824 / 2853$ & $28.90 \%(95 \% \mathrm{Cl}: 27.2-30.6)$ \\
\hline Female caregiver having had cytological screening & $307 / 2126$ & 14.40\% (95\%Cl: 13.0-16.0) \\
\hline \multicolumn{3}{|l|}{ Vaccines } \\
\hline Childhood vaccines & 2808/2994 & 93.80\% (95\%Cl: 92.9-.94.6) \\
\hline HPV second dose & $1933 / 3026$ & 63.80\% (95\%Cl: 62.13-65.6) \\
\hline HPV third dose & $1182 / 3026$ & $39.10 \%$ (95\%Cl: 37.3-40. 8) \\
\hline Mean time between second HPV dose and first dose & 33 days (SD 22.7) & \\
\hline Mean time between third HPV dose and second dose & 148 days (SD: 17.5) & \\
\hline Median time elapsed between first and last HPV dose & 175 days (IQR: 168-182) & \\
\hline
\end{tabular}


Table 2 Barriers/facilitators of full HPV vaccine regime

\begin{tabular}{llll}
\hline $\begin{array}{l}\text { Barriers/facilitators of full HPV } \\
\text { vaccine regimen }\end{array}$ & Odds Ratio & $95 \% \mathrm{Cl}$ & $P$ value \\
\hline $\begin{array}{l}\text { Administration of second } \\
\text { dose of HPV }\end{array}$ & 61.1 & $40.9-91.0$ & $<0.0001$ \\
HPV knowledge & 1.2 & $1.1-1.5$ & 0.008 \\
Male guardian & 1.14 & $1.0-1.3$ & 0.04 \\
Pap Smear test knowledge & 1.2 & $1.0-1.4$ & 0.08 \\
Childhood vaccine & 1.2 & $0.8-1.6$ & 0.4 \\
Religion & 1.1 & $0.4-2.5$ & 0.9 \\
Female guardian who had & 0.9 & $0.7-1.2$ & 0.6 \\
a Pap Smear test & & & \\
Differences in medians & & & 0.1 \\
$\quad \begin{array}{l}\text { Age } \\
\text { Distance }\end{array}$ & & & 0.01 \\
\hline
\end{tabular}

This research builds on limited research in Kenya on HPV vaccine acceptability for young girls and sought to explore factors associated with administration of the HPV vaccine, which is to our knowledge the first study to explore bottlenecks and facilitators for receiving 2 or 3 doses of HPV vaccine regimen. We have a vaccine uptake of $64 \%$ of two doses compared with other countries with less than $50 \%$. Kenya is considering a national HPV vaccine program through GAVI after a successful pilot in 2015 .

Our study revealed that only about $40 \%$ of school girls who had a first HPV dose got the third dose. Our low full uptake findings echo in Becker-Drep's study et al. [15] on HPV vaccine acceptability among Kenya women, where women expressed a strong preference for a vaccine that would require only one dose as opposed to a vaccine requiring three doses [15]. In malaria endemic settings like Western Kenya, uptake of three doses may be negatively affected hence the need for more studies on the effect of malaria on long term vaccine protection and dosing.

Our very strong statistically significant association observed between administration of the second HPV dose and third HPV dose within the six-month time period that was initially stipulated by the WHO before the 2 dose regimen was introduced in 2014 suggests that the majority of guardians who are favourable to a second dose of HPV vaccine are willing and also understand the necessity of having their daughters receive a third HPV dose.

Vermandere et al. qualitative study on implementation of HPV vaccine program in Eldoret in Eldoret by key stakeholders showed that caretakers and teachers poorly understood cervical cancer and linked it with modern lifestyle and nonconforming sexual behavior. And few had ever heard about the vaccination opportunity and felt uncomfortable to discuss cervical cancer. Teachers needed support from health care workers to address questions from parents and there was distrust towards HPV vaccines [33].

Masika et al. [34] found out that knowledge about HPV vaccine among school teachers was low to moderate but high vaccine acceptability hence may affect uptake. Empowering teachers to be vaccine champions in their community may be a feasible way of disseminating information about HPV vaccine and cervical cancer [34].

Distance was found to be a significant predictor of HPV vaccine uptake, which suggests that a different vaccine delivery strategy be envisaged. This observation also concurs with Vermandere et al. (2012) observations that acceptability of the HPV vaccine may not translate in higher HPV uptake, lest operational problems are addressed for parents.

As expected since the outcomes of interest were facilitators and barriers for HPV protection after 1st dose and not uptake of the first dose, it was anticipated that no significant association would then be found between Christian and Muslim religion. With less than $1 \%$ of the study population reporting a religion other Christianity, we expected a slightly higher percentage given that the Muslim population in our study setting is close to $10 \%$ further studies should be conducted to find out any relationship between religion and vaccine acceptability and uptake.

Intuitively, we would have expected female parents/ guardians to be significant predictor of three dose vaccine administration; however our results show that male parent/guardians were significantly associated with three dose regimen administration.

Also, whilst we observed a strong association between HPV knowledge and HPV vaccine uptake, unexpectedly, no statistically significant association was detected with undergoing Pap smear test by female parent/guardian, which may be indicative of the scarcity of information delivered to women receiving cytological screening on the necessity of HPV vaccine uptake for cervical cancer prevention.

\section{Strength and limitations}

The study's strength is that is our big sample size and that it is the first study to our knowledge that explores facilitators and barriers for HPV vaccine regimen uptake. However, our limitations include lack of data on potential known confounders, including meteorological conditions and time used to travel to the vaccine center as well as other potential unknown confounders which may not have been identified.

WHO guidelines 2014 and the CDC guidelines of 2016 have recommended 2 doses of HPV vaccine to girls less than 15 years old except those that are immunocompromised and HIV infected and 3 doses for those 
15 years and older, immunocompromised, HIV infected and this may hamper generalizability of our findings [7].

\section{Policy implications and research gaps}

Our findings highlighting the low HPV vaccine uptake has a number of policy implications which could be addressed within the future Kenyan National Cervical Cancer Prevention strategic Plan (https://www.k4health.org/ toolkits/Kenya-health-national-cervical-cancer-prevention-program-strategicplan-2012-2015. The observations derived from our study suggest that the plan could also promote screening for women so that they subsequently understand the importance of HPV vaccination and the completion of the recommended dose regimen. With research ongoing with respect to one dose HPV, implications on HIV, malaria/helminth infestation should be taken into consideration.

As distance to the clinic was found in our study to be a strong predictor of HPV vaccine uptake, a schoolbased vaccination, which has a track record of being more efficient [35], may be an alternative strategy providing that school attendance among girls reaches a certain threshold. Empowering teachers to be vaccine champions in their community may be a feasible way of disseminating information about HPV vaccine and cervical cancer and subsequently improve uptake.

Lastly, our current lack of knowledge of operational bottlenecks in Kenya for HPV vaccination uptake would benefit from qualitative studies to inform future quantitative survey tools. Qualitative studies are needed to illuminate our finding concerning why male guardians appear to be conducive to higher uptake of the HPV vaccine regimen.

\section{Conclusion}

Findings from this study suggested a need to consider school-based vaccination and more active education of caregivers to increase attainment of HPV vaccination regimen. Promoting screening among women so that they, in turn be targeted by an awareness campaign on the importance of HPV vaccination for cervical cancer prevention.

Qualitative methods are urgently needed to illuminate our study's quantitative findings and to inform future time series of cross-sectional surveys to reflect the trends of knowledge and barriers locally.

\section{Abbreviations}

ASRs: Age Standardized Rates; CDC: Centers for Disease Control and Prevention; FDA: Food and Drug Administration; GAP: GARDASIL Access Program; GAVI: Global Alliance for Vaccines and Immunization; HIV: Human Immunodeficiency Syndrome; HPV: Human Papilloma Virus; TOT: Training of Trainers; USA: United States of America; WHO: World Health Organization

\section{Acknowledgements}

We would like to thank Margaret Kabon for secretarial services and all the nurses involved with the administration of the vaccines headed by Sr Birgen.

\section{Funding}

Global Access Program and Merck for shipment and donation of the Gardasil Vaccine respectively. Merck did not provide direct financial support. Ghent University VRIL/Moi University Program supported the delivery of the vaccines to the girls.

\section{Availability of data and materials}

The datasets used and analysed during the current study are available from the corresponding author on request.

\section{Authors' contributions}

HM: Was involved in the application of the donation of the vaccines from Merck, primary conception and design of the study, acquisition of data, data analysis and interpretation of data. Submission of the study. He was also involved in drafting of manuscript and critical revision of the study. He has given final approval version for publication of this study and has agreed to be accountable for all aspects of the work related to the accuracy and integrity of the study. He wrote the paper SM: Was involved data analysis and interpretation of data. She was also involved in drafting of manuscript and revision of the manuscript. SW: Was involved in data analysis, interpretation of data, and revision of the manuscript. OM: Was involved in manuscript drafting, data analysis revision and literature search and manuscript revision EK: Was involved in promotion of the vaccination program, Literature search and manuscript revision EM: Was involved in manuscript design, literature search and revision. VN: Was involved in manuscript drafting, literature search and revision of the manuscript. HV: Was involved in Promotion of the vaccination program, literature search and manuscript revision DV: Data analysis, manuscript revision and data interpretation and guidance on this manuscript preparation. All authors have read and approved the manuscript.

\section{Ethics approval and consent to participate}

The HPV vaccination program was rolled out from May 2012 till March 2013 in Eldoret, Kenya. With support from the GARDASIL Access Program (GAP) through donation of Gardasil from Merck. Moi Referral and Teaching Hospital was able to vaccinate 3000 girls against cervical cancer. The vaccines were given for free in the hospital on Wednesdays and Saturdays. Promotion took place in ten randomly selected schools as to avoid over-demand in the community. Through this, 4000 eligible girls, i.e. girls from class 4 to 8 (9-14 years old), were targeted. Health care providers went to the schools to inform the teachers who were then asked to promote the vaccine among the students and their parents. The parents gave a written consent before each girl was vaccinated.

Written consent to the survey was obtained from all parents/guardians. Information collected was kept confidential by using numbers and codes. Ethical approval was obtained from the Ethics Committee of the Moi Teaching and Referral Hospital/Moi University School of Medicine (IREC). FAN: IREC 0001609.

\section{Competing interests}

The authors declare that they have no competing interests.

\section{Publisher's Note}

Springer Nature remains neutral with regard to jurisdictional claims in published maps and institutional affiliations.

\section{Author details}

${ }^{1}$ Moi University, Eldoret, Kenya. ${ }^{2}$ International Center of Reproductive Health, Ghent University, De Pintelaan 185 P3, 9000 Ghent, Belgium. ${ }^{3}$ CDC Foundation, Atlanta, Georgia, USA. ${ }^{4}$ Ghent University, Ghent, Belgium.

Received: 6 March 2017 Accepted: 17 April 2018

Published online: 11 May 2018

References

1. Ferlay J, Soerjomataram I, Ervik M, et al; International Agency for Research on Cancer. GLOBOCAN 2012 v1.0, Cancer Incidence and Mortality 
Worldwide: IARC Cancer Base No. 11. globocan.iarc.fr. Accessed 12 Dec 2013.

2. Nairobi Cancer Registry-NCR (2006); Cancer Incidence Report, Nairobi 2000-2002. Available from http://www.africacancer.org/downloads/ cancerincidenceReport-KEMRI

3. Muthoni MA, Ochieng OG, Mburugu KR, Samson N, Taratisio N, Rose G. Socio-demographic characteristics influencing uptake of screening for cervical Cancer in women aged 18-49 years in Imenti north sub-county, Meru County, Kenya. Science Journal of Public Health. 2016;4(2):94-9. https://doi.org/10.11648/j.sjph.20160402.13.

4. MOH: Ministry of Health: review of the 2004-2008 reproductive Health Research agenda proposed 2010-14 research agenda 2010. WHO/ICO information Centre on HPV and cervical Cancer (2011). HPV and related cancers in Kenya summary report retrieved on 31 January 2011. Online www.who.int/hpvcentre

5. WHO/ICO: Human Papilloma Virus and related cancers in Kenya. In ICO Information Centre on HPV and Cancer. Geneva: http://www.hpvcentre.net/ statistics/reports/KEN_FS.pdf (Accessed 31/07/2015).

6. Years of life lost as a measure of cancer burden on a national level O T Brustugun, B Møller, and Å Helland. Br. J.Cancer 2014; 111(5): 1014-1020. https://doi.org/10.1038/bjc2014364.

7. Human papillomavirus vaccines: WHO position paper, October 2014. http:// www.who.int/wer/2014/wer8943

8. Velde N, Boily M, Mélanie M, Franco E, Mayrand M, Kliewer E, Coutlée F, Laprise F, Malagón T, Brisson M. Population-level impact of the bivalent, quadrivalent and nonavalent human papillomavirus vaccines. Model- based analysis. J Natl Cancer Inst. 2012;104(22):1712-23.

9. Moodley I, Tathiah N, Mubaiwa V, Denny L. High uptake of Gardasil vaccine among 9-12-year-old schoolgirls participating in an HPV vaccination demonstration project in KwaZulu-Natal, South Africa. S Afr Med J. 2013;103: 313-7.

10. Ayissi CA, Wamai RG, Oduwo GO, Perlman S, Welty E, Welty T, Manga S, Ogembo JG. Awareness, acceptability and uptake of human papilloma virus vaccine among Cameroonian school-attending female adolescents. J Commun Health. 2012;37:1127-35.

11. Ladner et al:: Performance of 21 HPV vaccination programs implemented in low and middle-income countries, 2009-2013. BMC Public Health 2014 14: 670. https://doi.org/10.1186/1471-2458-14-670

12. Zimet GD, Liddon N, Rosenthal SL, Lazcano-Ponce E, Allen B. Chapter 24 psychological aspects of vaccine acceptability. Vaccine. 2006;24:S201-9. https://doi.org/10.1016/j.vaccine.2006.06.017.

13. Allen JD, Coronado GD, Williams RS, Glenn B, Escoffery C, Fernandez M, et al. A systematic review of measures used in studies of human papillomavirus (HPV) vaccine acceptability. Vaccine. 2010;28:4027-37. https://doi.org/10.1016/j.vaccine.2010.03.063 pmid: 20412875.

14. Lenselink CH, Gerrits MJ, Massuger L, van Hamont D, Bekkers R. Parental acceptance of human papillomavirus vaccines. Eur J Obstet Gynecol Reprod Biol 2008; 137: 103-107. https://doi.org/10.1016/j.ejogrb.2007.02.012 pmid: 17368910

15. Becker-Dreps S, Otieno WA, Brewer NT, Agot K, Smith JS. HPV vaccine acceptability among Kenyan women. Vaccine. 2010;28:4864-7. https://doi. org/10.1016/j.vaccine.2010.05.034. 20566394

16. Watson-Jones D, Mugo N, Lees S, Mathai M, Vusha S, Ndirangu G, Ross DA Access and attitudes to HPV vaccination amongst hard-to-reach populations in Kenya. PLoS One. 2015;10(6):e0123701. https://doi.org/10.1371/journal. pone.0123701.

17. Borkow G, Bentwich Z. Chronic parasite infections cause immune changes that could affect successful vaccination. Trends Parasitol. 2008;24:243-5.

18. Lehtinen M, Herrero R, Mayaud P, Barnabas R, Dillner J, Paavonen J. Studies to assess the long-term efficacy and effectiveness of HPV vaccination in developed and developing countries. Vaccine. 2006;24(Suppl 3):S3/233-41.

19. Walboomers $\mathrm{JL}$, et al. Human papillomavirus is a necessary cause of invasive cervical cancer worldwide. J Pathol. 1999;189:12-9.

20. Bosch FX, Lorincz A, Muñoz N, Meijer CJ, Shah KV. The causal relation between human papillomavirus and cervical cancer. J Clin Pathol. 2002; 55(4):244-65.

21. Cooper PJ, Espinel I, Paredes W, Guderian RH, Nutman TB. Impaired tetanusspecific cellular and humoral responses following tetanus vaccination in human onchocerciasis: a possible role for interleukin-10. J Infect Dis. 1998;178:1133-8.

22. Elias D, Wolday D, Akuffo $H$, Petros B, Bronner $U$, Britton S. Effect of deworming on human $T$ cell responses to mycobacterial antigens in helminth-exposed individuals before and after bacille Calmette-Guerin (BCG) vaccination. Clin Exp Immunol. 2001:123:219-25.

23. Elliott AM, Mawa PA, Webb EL. Effects of maternal and infant coinfections, and of maternal immunisation, on the infant response to BCG and tetanus immunisation. Vaccine. 2010;29:247-55.

24. Mirza NB, Wamola IA, Estambale BA, Mbithi E, Poillet M. Typhim vi vaccine against typhoid fever: a clinical trial in Kenya. East Afr Med J. 1995;72:162-4.

25. Patriarca PA, Wright PF, John TJ. Factors affecting the immunogenicity of oral poliovirus vaccine in developing countries: review. Rev Infect Dis. 1991; 13:926-39.

26. Sabin EA, Araujo MI, Carvalho EM, Pearce EJ. Impairment of tetanus toxoid specific Th1-like immune responses in humans infected with Schistosoma mansoni. J Infect Dis. 1996;173:269-72.

27. Triki H, Abdallah MV, Ben Aissa R. Influence of host related factors on the antibody response to trivalent oral polio vaccine inTunisian infants. Vaccine. 1997;15:1123-9.

28. Kenya Malaria Indicator Survey (2015). https://www.knbs.or.ke/kenya-malariaindicator-survey-2015/.

29. Ministry of Health (2014). https://www.ncbi.nlm.nih.gov/pmc/articles/ PMC5146872/.

30. WHO World Malaria Report 2016. http://www.who.int/malaria/publications/ world-malaria-report-2016/en/

31. Brown J, Baisley K, Kavishe B, John Changalucha J, Andreasenc A, Mayaudc P, Gumodoka B, Kapiga K, Hayes R, Watson-Jones D. Impact of malaria and helminth infections on immunogenicity of the human papillomavirus-16/18 AS04-adjuvanted vaccine in Tanzania. Vaccine. 2014;32:611-7.

32. Brooker S, Kabatereine NB, Gyapong JO, Stothard JR, Utzinger J. Rapid mapping of schistosomiasis and other neglected tropical diseases in the context of integrated control programmes in Africa. Parasitology. 2009;136: 1707-18.

33. Vermandere H, Naanyu V, Degomme O, Michielsen K. Implementation of an HPV vaccination program in Eldoret, Kenya: results from a qualitative assessment by key stakeholders. BMC Public Health. 2015;15:875. https://doi. org/10.1186/s1 2889-015-2219-y.

34. Masika MM, Ogembo JG, Chabeda SV, Wamai RG, Mugo N. Knowledge on HPV vaccine and cervical Cancer facilitates vaccine acceptability among school teachers in Kitui County, Kenya. PLoS One. 2015;10(8):e0135563. https://doi.org/10.1371/journal.pone.0135563.

35. Paul P, Fabio A. Literature review of HPV vaccine delivery strategies: considerations for school- and non-school based immunization program. Vaccine. 2014;32:320-6.

\section{Ready to submit your research? Choose BMC and benefit from:}

- fast, convenient online submission

- thorough peer review by experienced researchers in your field

- rapid publication on acceptance

- support for research data, including large and complex data types

- gold Open Access which fosters wider collaboration and increased citations

- maximum visibility for your research: over $100 \mathrm{M}$ website views per year

At BMC, research is always in progress.

Learn more biomedcentral.com/submissions 